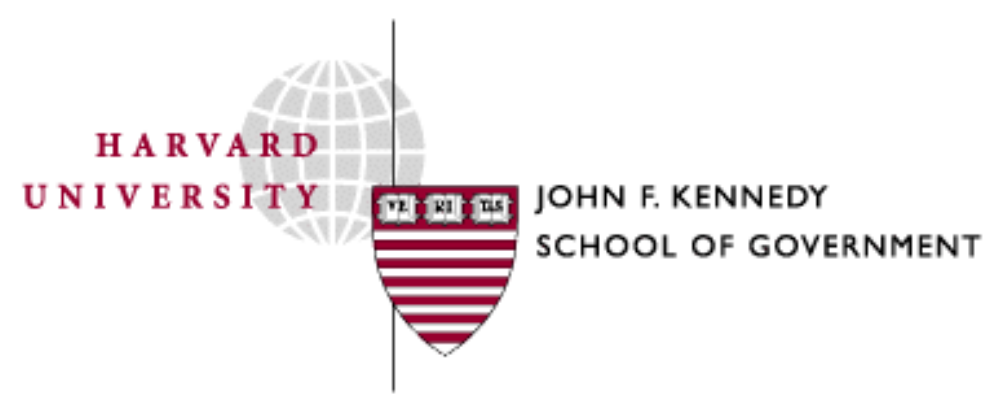

Faculty Research Working Papers Series

\title{
Mechanisms for Enhancing the Credibility of an Adviser: Prepayment and Aligned Incentives
}

\section{Anthony G. Patt, Hannah Riley Bowles, and David W. Cash \\ March 2006 \\ RWP06-010}

This paper can be downloaded without charge from: http://ksgnotes1.harvard.edu/Research/wpaper.nsf/rwp/RWP06-010

or

The Social Science Research Network:

http://ssrn.com/abstract $=896147$

The views expressed in the KSG Faculty Research Working Paper Series are those of the author(s) and do not necessarily reflect those of the John F. Kennedy School of Government or Harvard University. Copyright belongs to the author(s). Papers may be downloaded for personal use only. 
Mechanisms for Enhancing the Credibility of an Adviser:

Prepayment and Aligned Incentives

\author{
Anthony G. Patt Boston University
}

Hannah Riley Bowles Harvard University

David W. Cash

Massachusetts Executive Office of Environmental Affairs

(Forthcoming in the Journal of Behavioral Decision Making)

Keywords: Advice, judge adviser systems, trust, sustainable development

\begin{abstract}
We tested the effectiveness of prepayment for advice and aligned incentives as mechanisms for enhancing trust in unfamiliar advisers in decision-making under uncertainty. Participants were low-income Zimbabweans who played two rounds of the Monty Hall three-door game. In round 1, participants who purchased advice were significantly more likely to follow advice for how to win the game than were participants who received free advice. In round 2, the apparent effectiveness of advisers' suggestions in round 1 moderated participants' propensity to follow advice. If the round 1 advice appeared wrong, the credibility enhancing benefits of prepayment diminished. If the advice appeared right, the benefits of prepayment maintained. Hypotheses with regard to the benefits of aligned incentives received only weak support.
\end{abstract}




\section{Mechanisms for Enhancing the Credibility of an Adviser:}

Prepayment and Aligned Incentives

When decision-makers receive advice, they must decide how much to trust that advice relative to their own opinion. In situations in which the credibility of the person giving advice is uncertain, decision-makers' trust in the social context in which they receive advice can substitute for personal trust in the advisers themselves. Research has shown that the roles that advisers and decision-makers play in a social system may influence the level of trust between them, the acceptability of the advice that is offered, and the final outcome of the decision (Yaniv, 2004a).

This question of how the social context of advice giving influences the relationship between advisers and decision-makers is relevant to many real-world problems (Sniezek \& Buckley, 1995), because as our society has become more complex the dissemination of expert advice has become increasingly detached from personal relationships (Earle \& Cvetkovich, 1995). Public health experts espouse strategies for stemming the tide of infectious disease. Security experts and emergency responders attempt to educate the public about effective strategies for preventing and responding to man-made and natural disasters. Environmental scientists advocate for reform of unsustainable resource management practices.

The challenge of enhancing the credibility of expert advice becomes even more acute in situations in which legitimizing social institutions are weak and decision-makers lack access to the educational resources to judge the quality of the advice they are receiving. Such circumstances are common, for instance, to policy making in many developing countries (Cash, Borck, \& Patt, in press). Under these conditions, valuable advice often goes unheeded in spite of the need for assistance, because decision-makers have little basis upon which to judge the credibility of the unfamiliar advisers (Lemos, Finan, Fox, Nelson, \& Tucker, 2000).

We conducted an experiment in which we examined how the manipulation of the adviser-decision-maker relationship would influence decision-makers' willingness to follow an unfamiliar adviser's suggestions. We tested the effects of the adviser-decisionmaker relationship in a situation of decision-making under uncertainty, so that even the best advice (i.e., the dominant solution given the payoff structure) would not guarantee a positive outcome. In this way, we were also able to explore whether the credibilityenhancing influence of the adviser-decision-maker relationship would remain robust even when good advice did not guarantee positive payoffs.

We conducted the experiment in Zimbabwe. This helped us to address the common critique that experimental research has little relevance to real-word decisionmaking because the stakes are so low (Camerer \& Hogarth, 1999; Hertwig \& Ortman, 2001). The value of the U.S. dollar in Zimbabwe made it financially feasible to raise the decision-making stakes to a level equivalent to half of the daily per capita income. Conducting the experiment in Zimbabwe also enabled us to extend the generalizability of the existing research on adviser-decision-maker relationships (e.g., Sniezek, Schrah, \& Dalal, 2004) to a developing country context and, as discussed in the conclusion, to test hypotheses about advice-giving in developing countries that had previously only been explored through case research (Cash et al., 2001).

When Do Decision-makers Follow Advice? 
One way in which decision-makers discern the credibility of advice is to judge its apparent value relative to their own knowledge, as well as whether it appears internally consistent. When information from one or more sources appears inconsistent, people are less likely to use it to inform their decision-making (Slovic, 1966; cf. Sniezek \& Buckley, 1995). Decision-makers are more likely to use outside advice when their own knowledge of a subject is limited (Harvey \& Fischer, 1997), and look to the relevant qualifications of potential advisers to discern the reliability of their advice. For instance, when purchasing a used car, decision-makers are significantly more likely to rely on advisers with higher levels of mechanical expertise (Birnbaum, Wong, \& Wong, 1976). Decision-makers also form judgments about the credibility of advisers based on repeated interactions with them and the observed quality of the advice that they provide (Yaniv, 2004b; Yaniv \& Kleinberger, 2000). If decision-makers are in a poor position to assess the credibility of the adviser or the advice itself, they may take their cues from the social context in which the advice is provided.

One potential mechanism for enhancing decision-makers' trust in unfamiliar advisers is prepayment. Sniezek et al. (2004) found that decision-makers were more likely to use information when they had paid for it, particularly if the payment took place before rather than after they used the information. Sniezek and colleagues argue that prepayment for advice creates a form of "psychological contract" (Robinson, 1996), giving the adviser an implicit obligation to provide high quality information. Prepayment may also enhance decision-makers' perceptions of the value of advice simply because they presume more expensive goods are of higher quality (Bagwell \& Riordan, 1991; Fluet \& Garella, 2002; Teas \& Agarwal, 2000). It is also possible that decision-makers are more motivated to use purchased advice because they value goods more if they already own them ("endowment effect") (Kahneman, Knetch, \& Tversky, 1990; Kahneman \& Tversky, 1979) and/or are loath to discard a good for which they have paid ("sunk cost fallacy") (Thaler \& Johnson, 1990).

These alternative explanations for the effectiveness of prepayment as a mechanism for enhancing trust in unfamiliar advisers could have distinct implications if the advisers and decision-makers are engaged in more than one round of interaction and the decisions are made under uncertainty (i.e., good advice cannot guarantee a positive outcome). If prepayment engenders the perception of a psychological contract between the adviser and decision-maker, then the decision-maker may be more inclined to perceive advice that does not pay off as a breach of trust. In such a case, the trustenhancing benefits of prepayment may not carry over to future interactions if past advice did not prove beneficial. Alternatively, if purchasing advice merely enhances the perceived value of the advice, then prepayment should still be more influential than free advice, even if the advice did not pay off in the past.

Another mechanism that decision-makers may rely upon when they lack the information to judge the quality of their advisers' work is aligned incentives. The problem of a decision-maker trying to assess the credibility of advice from an unfamiliar adviser may be conceived of as a form of principal-agent problem, to the extent that the decision-maker lacks the ability to assess the quality of advice being provided and cannot discern its quality from the outcome of the decision alone (e.g., in the case of decisionmaking under uncertainty) (Arrow, 1985). One way to address this information problem is for decision-makers to align the interests of their advisers with their own, so that their 
advisers share in the risks of the decision and are thereby motivated to provide the best advice possible (Arrow, 1985; Laffont \& Martimort, 2002). We predict that decisionmakers will be more likely to follow the suggestions of advisers whose interests are aligned with their own than those of advisers who do not share a stake in the outcome (e.g., an adviser offering free advice). Even if the advisers' suggestions do not pay off initially, either because the advice was wrong or because the decision-making problem had a stochastic outcome, the decision-makers should still have more confidence in the word of advisers with aligned incentives, because they know that it is in their advisers' best interests to suggest the course of action with the highest likely payoff.

$$
\text { Study }
$$

We tested the effects of prepayment for advice and aligned incentives as mechanisms for enhancing trust in unfamiliar advisers in a situation of decision-making under uncertainty, in which advisers provided advice over two rounds of interaction. The experimenter revealed at the end of each round whether following the advisers' suggestions would have produced a positive payoff for the decision-maker. We tested whether prepayment for advice and aligned incentives would enhance decision-makers' propensity to follow advice (as compared to simple advice given by an adviser who receives no compensation and has no stake in the outcome) in the first round of decisionmaking and whether the experience of watching the advice fail or succeed in the first round would affect the propensity to follow the advice in the second round of decisionmaking.

Consistent with the results of previous research by Sniezek et al. (2004), we predicted that decision-makers would be more likely to follow purchased advice than simple advice in the first round of decision-making. If the advisers' suggested course of action in round 1 would have produced a positive payoff, then we predicted that the credibility-enhancing advantage of prepayment for advice over simple advice would be maintained in round 2. The alternative interpretations of Sniezek et al.'s findings suggested competing hypotheses for how decision-makers would respond to prepayment in round 2 if their advisers' suggestions in round 1 would not have produced a positive payoff. Following Sniezek et al.'s theory that prepayment for advice engenders the perception of a psychological contract between the adviser and decision-maker, we predicted that observing that the adviser's suggested course would not have worked in round 1 would produce a greater decline in the use of the advisers' suggestions under prepayment than under simple advice in round 2 . This is because the decision-makers would feel more betrayed by an adviser they had paid than by an adviser offering free suggestions. Alternatively, if, as described above, decision-makers just perceived purchased advice to be more valuable than free advice, then decision-makers should still be more inclined follow purchased advice than simple advice after seeing that the advisers' suggested course of action would not have paid off in round 1.

We predicted that decision-makers would be more likely to follow the suggestions of advisers with aligned incentives than simple advice in round 1 . We predicted that the credibility-enhancing effect of aligned incentives as compared to simple advice would remain robust in round 2 , even if the decision-makers observed that the advisers' suggestions would not have paid off in round 1. This is because decision-makers would perceive advisers with aligned incentives to have a shared stake in producing the best possible outcome in round 2. 
While we predicted effects of prepayment for advice and aligned incentives relative to simple advice, we made no predictions about their performance relative to each other. In addition to the three advice conditions, we also tested a control condition, in which no advice was offered. We conducted the control condition in order to examine whether the performance of our subject pool in the decision-making task was similar to that of other subject pools that have been observed engaging in the same decision-making task and to provide a baseline for behavior in the absence of any advice. We made no prediction with regard to how much influence simple advice would have on the participants' behavior relative to the control condition.

\section{Method}

\section{Participants}

Participants were 359 adults (182 male, 177 female) from poor residential neighborhoods of the two largest cities of Zimbabwe, Harare and Bulawayo. The per capita income in these neighborhoods was less than $\$ 2$ per day. They received approximately US $\$ 0.50$ in local currency for participating plus the opportunity to earn more money based on their performance in the decision-making exercise.

\section{Procedure}

All participants played two rounds of the three-door game, first popularized on a 1970's television show hosted by Monty Hall (Friedman, 1998). In each round of the game, the experimenter showed the participant a panel with three closed doors (Doors 1, 2, and 3), which appeared identical in all respects. The experimenter explained that there was a cash prize behind one of the three doors, and instructed the participant to make an initial selection of which door to open. Once the participant had made an initial selection, the experimenter provided the participant a hint as to which door contained the prize by opening one of the other two doors that the participant had not selected and that did not contain the prize. After providing the hint (i.e., opening the empty door), the experimenter gave the participant the choice of whether to stay with the original door already selected or to switch to the other closed door. Once the participant indicated which door the experimenter should open, the experimenter opened that door and revealed whether the participant had won the prize.

The math behind the three-door game is simple yet deceptive. There is a one-third chance that the participant's initial selection is the winning door. Therefore, when participants remain with their initial selection as their final choice, they win the prize with probability one-third. If they switch to the one remaining door after the hint, they win with probability two-thirds. The three-door game is a well-established choice anomaly. Past studies have shown that most people erroneously believe the two remaining doors after the hint are equally likely to be the winning door, and that very few people switch doors (Franco-Watkins, Derks, \& Dougherty, 2003; Krauss \& Wang, 2003; Tubau \& Alonso, 2003). Across different cultures, fewer than $20 \%$ of participants switch doors the first time they play the game, and only after many rounds does the percentage of people switching rise past fifty (Granberg, 1999; Granberg \& Brown, 1995).

In our control condition, participants did not receive any advice about whether to switch doors after the hint had been offered. In the other three conditions, a third person, who served as an "adviser" to the participant, suggested to the participants that they should switch doors after the hint. The advice never varied, and consisted of the following script: 
I do not know which door will win, but I have watched this game played many times. Switching doors will not always win, but you have a much higher chance of winning if you switch doors rather than stay with your initial pick. I suggest that you switch doors, though of course you are free to do otherwise.

The experimenter ran the game using a stack of cardboard panels each containing the three doors on their front side. The front side of the panels looked identical. On the backside of each panel was written the winning door. Which door would win varied uniformly among the panels, and the experimenter showed this to the participants by opening doors on several panels before shuffling the stack. For each play of the game the experimenter selected a panel at random from the stack, looked at the back of the panel to see which door would win, and showed only the front side to both the participant and the adviser. In this way, it was clear that the adviser did not know which was the winning door.

The adviser was always a well-spoken, male, secondary school graduate in his mid 20's who was recruited from the local area, but was unfamiliar to the participants. Four different men played the role of adviser across the experimental sessions. (There were no significant adviser effects on the participants' propensity to switch doors in any of the treatment groups, $p s>$.27.) Prior to his participation in the game, each adviser had the opportunity to play and observe the game many times. The experimenter explained to the adviser why there was a higher likelihood of success after switching doors until it was evident that the adviser believed and understood the advice he was giving. The experimenter informed the advisers that, in some cases, they would make additional money during the game, because they would receive a payment equal to that of the participant or because the participant would pay them for their advice. The advisers were blind to the experimental hypotheses.

The experimenter recruited subjects in each neighborhood by driving into the neighborhood and parking in a visible location, and asking all adult passersby if they would like to participate in a short psychological study for which they would receive compensation. The experimenter told the participants that the purpose of the study was to observe how people make choices. In each location, the experimenter stopped recruiting as soon as there was a group of 20 participants. Participants played one at a time. Those waiting to play stood in a group out of sight and sound of the participant playing and out of contact with those who had already played.

Each participant received an initial payment in local currency worth about US\$0.50. Before playing the game with each participant, the experimenter described the entire procedure of the three-door game and explained that the participant would be playing the game two times. For each round that the participants won, they received an additional cash payment of the same amount as their initial payment (US\$0.50).

In all conditions, the experimenter introduced the adviser immediately after giving participants their initial cash payment, and before telling participants how the game would work. In the control condition, the experimenter introduced the adviser as a man from the local community who was working with the experimenter for the day, but the man provided no additional information about the game. In the three treatment conditions, the experimenter introduced the adviser as a man from the local community who was working with the experimenter for the day and who would help the participants 
win the game by providing useful advice. The experimenter told participants that the adviser had received training in the game and the best way to win it, and had observed many people play the game. The adviser then introduced himself by name, and confirmed that the preceding statements about him were correct.

\section{Conditions}

The experimenter randomly assigned participants to play two rounds of the game under one of four conditions: control, simple advice, aligned incentives or prepayment. In the control condition $(N=51)$, participants received no advice about whether to switch doors. In the simple advice condition $(N=105)$, the experimenter did not tell participants any additional information (beyond the previous statement that the adviser was working with the experimenter for the day) about how much and under what conditions the adviser was being paid. In the aligned incentives condition $(N=56)$, the experimenter told the participants that their adviser would receive a payment after each play of the game equal to that of the participant. When the participant won the game, the experimenter immediately paid both the participant and the adviser the same amount of prize money. In the prepayment condition $(N=61)$, the experimenter gave the participant the opportunity to pay the adviser $10 \%$ of the initial cash payment (about US\$0.05) before round 1 to advise them during both rounds the game. Those who elected to pay ( $N$ $=47)$ received advice in both rounds, and those who elected not to pay $(N=14)$ received no advice.

\section{Round 1 Experience}

After Round 1, about two-thirds of the participants (64\%) observed that switching doors did or would have worked ("switching worked"), resulting from their initial selection randomly matching one of the two losing doors. Likewise, about one-third (36\%) initially selected the winning door by chance, and thereby observed that switching doors did not or would not have worked ("switching did not work"). If the participant received advice to switch doors, then observing switching doors (not) to work was synonymous with observing the advice (not) to work.

\section{Round 1}

$$
\text { Results }
$$

Figure 1 displays the rates of switching in the first round of play. The rate of switching in the control condition $(P=.22)$ was comparable to the average rate of switching in other experiments using the three-door game. There was no significant difference in the rate of switching between the control condition and the simple advice condition $(P=.27)$, 2

$\chi(1, N=156)=0.48, p=.49$. Contrary to our predictions, there was also no significant difference in the rate of switching between the simple advice condition and the aligned incentives condition $(P=.20), \chi(1, N=171)=1.08, p=.30$.

As predicted, the overall rate of switching in the prepayment condition $(P=.48)$ was significantly higher than in the simple advice condition, $\chi^{2}(1, N=166)=7.46, p<.01$. (This overall rate of switching includes both those who agreed and those who refused to purchase advice.) The overall rate of switching in the prepayment condition was also significantly higher than the rate of switching in the aligned incentives condition, $\chi(1, N$ $=127)=11.10, p=.001$. As shown in the two right-hand bars of Figure 1, participants in the prepayment condition split into two categories: 47 of the 61 participants in the prepayment condition agreed to purchase the advice (prepayment [yes]), and the 
remaining 14 refused and received no advice (prepayment [no]). The rate of switching among those who agreed to purchase the advice $(P=.60)$ was 2.2 times higher than in the simple advice condition, $\chi^{2}(1, N=152)=15.11, p<.001$. Whereas the rate of switching among those who refused to purchase advice $(P=.07)$ was statistically indistinguishable from the rate of switching in the control condition, $\chi^{2}(1, N=65)=1.52, p=.22$. We omit consideration of those who refused to purchase advice from analyses of round 2 behavior. Round 2

Experience Effects. As described above, the results of round 1 randomly assigned participants into two experience groups: those who observed switching doors work and those who observed switching doors not work. Figure 2 displays the rate of switching doors in round 2 by condition and round 1 experience. Observing switching doors work (vs. not work) in round 1 had no significant effect on the rate of switching in round 2 in the control condition, switching work $N=36, P=.33$, switching not work $N=15, P=$ $.20, \chi(1, N=51)=.91, p=.34$. The experience of observing switching doors work (vs. not work) in round 1 did, however, significantly increase the rate of switching in round 2 in each of the conditions in which participants received advice. In the simple advice condition, those who observed switching work $(N=65)$ followed the advice at a rate of $P$ $=.43$, whereas those who observed switching not work $(N=40)$ followed the advice at a rate of $P=.15, \chi^{2}(1, N=105)=8.92, p=.003$. In the aligned incentives condition, the rate of switching $(P=.62)$ among those who observed switching work $(N=37)$ was significantly higher than the rate of switching among those $(N=19)$ who observed switching not work $(P=.28), \chi^{2}(1, N=56)=7.80, p=.005$. The rate of switching $(P=$ $.68)$ among those participants who obtained prepaid advice and observed switching work $(N=34)$ was also higher than the rate of switching among those participants $(N=13)$ who purchased advice and then observed switching not work $(P=.23), \chi(1, N=47)=$ $7.56, p=.006$.

Observed Switching Work. While participants in the simple advice condition were significantly more likely to switch in round 2 after observing switching doors work (vs. not work) in round 1 , those in the simple advice condition who observed switching work were still no more likely to switch in round 2 that were those in the control condition who observed switching work, $\chi^{2}(1, N=101)=0.92, p=.34$. Participants in the aligned incentives condition who observed switching work in round 1 were only marginally significantly more likely to switch doors in round 2 than were participants in the simple advice condition who observed switching work, $\chi^{2}(1, N=102)=3.44, p=.06$. As predicted, participants who purchased advice and who observed switching work in round 1 were significantly more likely to switch doors in round 2 than were participants in the simple advice condition who observed switching work, $\chi(1, N=99)=5.40, p=.02$. Observed Switching Not Work. Participants in the simple advice condition who observed switching not work in round 1 were no more likely to switch that those in the control condition who observed switching not work, $\chi^{2}(1, N=55)=0.20, p=.66$. There was no significant difference in the rate of switching in round 2 between participants in the aligned incentive condition who observed switching not work and those in the simple 
advice condition who observed switching not work, $\chi^{2}(1, N=69)=1.65, p=.20$. There was also no significant difference in the rate of switching in round 2 between participants who purchased advice and who observed switching not work and those in the simple advice condition who observed switching not work, $\chi^{2}(1, N=53)=0.45, p=.50$. Comparing Round 1 and Round 2

Figure 3 displays the differences in the proportions of participants who switched in rounds 1 and 2 (round $2 P$ - round $1 P$ ), broken down by round 1 experience and condition. In order to account for the lack of independence between the participants' decisions to switch in rounds 1 and 2, we used the formula for the analysis of matched pairs of binary data to generate the standard error for each difference in proportions, as suggested by Agresti (1996). We calculated the significance of difference-in-difference comparisons from the standard errors and differences in proportions.

As illustrated in Figure 3, when the decision-makers observed the advice to work in round 1 , the aligned incentives manipulation produced a significantly greater increase in the rate of switching between rounds 1 and 2 than did simple advice, $z=1.98, p<.05$. When the decision-makers observed the advice not to work in round 1, the aligned incentives manipulation had no significant effect on the relative rate of switching between rounds 1 and 2 as compared to the simple advice condition, $z=1.47, p=.14$. This suggests that the aligned incentives manipulation did have a significantly positive effect in terms of enhancing the credibility of the unfamiliar adviser over time (as compared to simple advice), but only when his advice was also reinforced by a positive track record.

Round 1 experience had different implications for the behavior of decisionmakers who purchased advice. When the decision-makers observed the advice to work, there was a comparable increase in the rate of switching between rounds 1 and 2 in the prepayment (yes) and simple advice conditions, $z=0.13, p=.90$. However, when the decision-makers observed the advice not to work, the decline in the switching rate between rounds 1 and 2 was significantly greater when the participants had purchased the advice than when they had received simple advice, $z=2.99, p<.01$. These results suggest that the decision-makers were more rejecting of failed advice for which they had paid than of failed advice that was free.

\section{Discussion}

The results of our experiment build upon and extend previous research on the influence of adviser-decision-maker relationships on the credibility of expert advice. They replicate the effects of prepayment for advice under high stakes and with a nonindustrialized country population. By tracking the receptiveness to advice over two periods, they also illuminate the how vulnerable credibility-building mechanisms, such as prepayment, can be when even the best advice cannot guarantee positive results. Finally, these results make an important contribution to the largely qualitative literature on advisory institutions and technology transfer. By a conducting an experiment in a field setting, we provide empirical evidence that, controlling for the quality of advice and decision-making stakes, adviser accountability does influence the use of advice in developing country contexts.

In the initial round of decision-making under uncertainty, we found that prepayment for advice significantly enhanced the credibility of advice from an unfamiliar adviser. Participants who had the opportunity to purchase advice were significantly more 
likely to follow their advisers' suggestions than were participants who received free advice - regardless of whether the adviser shared a stake in the outcome of the decision or not. Contrary to expectations, giving the adviser a stake in the outcome of the decision (aligned incentives) produced no significant increase in the use of advice as compared to simple free advice. Interestingly, decision-makers who received simple advice (free advice, no stakes) behaved no differently than decision-makers who received no advice.

In the second round of decision-making under uncertainty, we found that the decision-makers' initial round experiences had important implications for the use of advice in subsequent decisions. When the advisers' suggested course of action in round 1 appeared wrong (i.e., it would not have produced a positive payoff based on the outcome of the game), there was no significant difference in the use of advice across conditions. The credibility enhancing benefits of prepayment were lost. Participants who purchased advice and then observed that advice not to work in round 1 exhibited a significant decline in the use of the advice in round 2. When the advisers' suggested course of action in round 1 appeared right (i.e., it would have produced a positive payoff based on the outcome of the game), the credibility enhancing advantages of prepayment were maintained: those participants who purchased advice were significantly more likely to follow their advisers' suggestions in round 2 than were participants who received simple advice.

One noteworthy limitation to the analysis of the round 2 results was that we limited comparisons between the prepayment for advice and simple advice conditions to the two-thirds of participants in the prepayment condition who had actually purchased advice. There was no meaningful comparison to make between the round 1 experience of participants who did not purchase advice (and, therefore, never heard the advice) and those participants who received free advice. Further investigation is needed to explore the implications of voluntary vs. compulsory prepayment relationships.

Contrary to expectations, the hypothesized benefits of aligned incentives for enhancing the credibility of unfamiliar advisers received only weak support. Aligned incentives never significantly outperformed simple advice over the course of the two rounds. However, when the advice appeared correct in round 1, the rate of increase in the use of the advisers' suggestions between rounds 1 and 2 was higher when the adviser had aligned incentives than when the adviser gave simple advice. This suggests that, when coupled with a positive track record, giving advisers a stake in the outcome of the decision might enhance their credibility over time. Future research is warranted to test the effectiveness of both prepayment for advice and aligned incentives over longer rounds of decision-making under uncertainty, in order to explore more deeply how chance-based outcomes moderate the credibility of unfamiliar advisers.

The results of the first round of decision-making support the findings of previous research by Sniezek et al. (2004), which showed that decision-makers were more likely to use advice for which they had paid. The current research extends the work of Sniezek and colleagues in a couple of important respects. First, by testing the effectiveness of prepayment for advice over two rounds of decision-making under uncertainty, we were able to demonstrate the vulnerability of this intervention to chance-based outcomes. Perhaps, because of a perceived betrayal of the psychological contract between decisionmakers and advisers under prepayment, the decision-makers who had paid for advice (as compared to those who received free advice) were significantly more rejecting of advice 
in round 2 that did not appear correct in round 1. There are few important, real-world decisions for which expert advisers can guarantee positive results from their proposed courses of action. Our results suggest that future research should explore mechanisms for improving the robustness of the credibility enhancing benefits of the prepayment mechanism over multiple rounds of decision-making under uncertainty.

This research also contributes to the work on learning in decision-making in the Monty Hall three-door game. Friedman (1998), for instance, tested several manipulations designed to promote learning over multiple rounds of the game, and found that offering decision-makers conflicting advice that included the correct explanation of the game significantly increased rates of switching by $13 \%$. More recently, Slembeck \& Tyran (2004) showed that manipulating group dynamics (i.e., introducing majority rule and competition among decision-makers) significantly increased the rate of switching. Combining majority rule decision-making with inter-group competition elevated switching rates to nearly $100 \%$ after several rounds. These studies and the current work demonstrate the importance of social interaction as well as experience for enhancing the effectiveness of decision-making under uncertainty.

One potentially important dimension of the advice-giving relationship that should be explored is the perceived social relationship between the advisers and decisionmakers. Previous research has shown that decision-makers infer reliability from social signals, such as common membership in a small social group, even when the perceived relationship is unrelated to the decision for which trust is required (Burnham, McCabe, \& Smith, 2000; Earle \& Cvetkovich, 1999). In a case study among pizza delivery firms, for example, researchers found that people were more likely to accept advice from other business organizations that had similar corporate strategies (Darr \& Kurtzberg, 2000). In a car-buying experiment, Birnbaum and Stegner (1979) found that perceived friendship ties between the expert adviser and the seller or buyer influenced receptivity to the advice. They also found that the more expert the adviser appeared to be, the more decision-makers took the potential bias in favor of the expert's friend into the account. In the current research, we recruited men from the local community to act as advisers. It remains open to question whether manipulating aspects of the advisers' identities (e.g., sex, race, political or institutional affiliation, or place or origin) would engender different levels of confidence in their suggestions.

Finally, this line of research has important implications for enhancing the credibility of expert advice in real-world situations. Case researchers have observed in a wide variety of field situations that those advisory groups that are directly accountable to the decision-makers, or have a strong financial incentive to provide high quality information, tend to be more readily trusted and are ultimately more effective than those lacking such accountability or incentives (Cash, et al., in press; Cash \& Moser, 2000; Guston, 1999, 2001; Patt, 2001). These studies have identified several factors that promote direct accountability, including the purchase of advice by decision-makers and institutional connections between decision-makers and advisors. These field studies have, however, also typically shown that in situations in which incentives are aligned and advisers are accountable to the decision-makers, the quality of the information provided is higher: the advisers tailor their message more closely to the actual decisions faced by their audience (Cash, 2000; Clark, Mitchell, Cash, \& Alcock, in press). It has been difficult, therefore, to discern from the existing field research the extent to which it is 
social context of the advice-giving relationship or the quality of the advice itself that enhanced the credibility of advisers in these contexts. By complementing field research with experiments conducted with practitioner-relevant populations, we can gain deeper insights into how experts may most effectively structure their advice-giving relationships to maximize their potential influence.

\section{References}

Agresti, A. (1996). An introduction to categorical data analysis. New York: John Wiley \& Sons.

Arrow, K. (1985). The economics of agency. In J. Pratt \& R. Zeckhauser (Eds.), Principals and agents: the structure of business. Boston: Harvard Business School Press.

Bagwell, K., \& Riordan, M. (1991). High and declining prices signal product quality. American Economic Review, 81(1), 224-239.

Birnbaum, M. H., \& Stegner, S. (1979). Source credibility in social judgment: bias, expertise, and the judge's point of view. Journal of Personality and Social Psychology, 37, 48-74.

Birnbaum, M. H., Wong, R., \& Wong, L. K. (1976). Combining information from sources that vary in credibility. Memory and Cognition, 4(3), 330-336.

Burnham, T., McCabe, K., \& Smith, V. L. (2000). Friend-or-foe intentionality priming in an extensive form trust game. Journal of Economic Behavior and Organization, 43, 57-73.

Camerer, C., \& Hogarth, R. M. (2000). The effects of financial incentives in experiments: A review and capital-labor-production framework. Journal of Risk and Uncertainty, 19(1-3), 7-42.

Cash, D. (2000). Distributed assessment systems: an emerging paradigm of research, assessment, and decision-making for environmental change. Global Environmental Change, 10(4), 241-244.

Cash, D., Borck, J., \& Patt, A. G. (in press). Countering the 'loading dock' approach to linking science and decision making: a comparative analysis of ENSO forecasting systems. Science, Technology, and Human Values.

Cash, D., \& Moser, S. (2000). Linking global and local scales: designing dynamic assessment and management processes. Global Environmental Change, 10(2), 109-120.

Clark, W., Mitchell, R., Cash, D. W., \& Alcock, F. (in press). Information as influence: how institutions mediate the impact of scientific assessments on global environmental affairs. In W. Clark \& R. Mitchell \& D. W. Cash \& F. Alcock (Eds.), Global environmental assessments: information, institutions, and influence. Cambridge, MA: MIT Press.

Darr, E., \& Kurtzberg, T. (2000). An investigation of partner similarity dimensions on knowledge transfer. Organizational Behavior and Human Decision Processes, 82(1), 28-44.

Earle, T. C., \& Cvetkovich, G. T. (1995). Social trust: toward a cosmopolitan society. Westport, Connecticut: Praeger.

Earle, T. C. \& Cvetkovich, G. T. (1999). Social trust and culture in risk management. In G. Cvetkovich \& R. Löfstedt (Eds.), Social trust and the management of risk (pp.9-21). London: Earthscan. 
Fluet, C., \& Garella, P. (2002). Advertising and price as signals of quality in a regime of price rivalry. International Journal of Industrial Organization, 20, 907-930.

Franco-Watkins, A., Derks, P., \& Dougherty, M. (2003). Reasoning in the Monte Hall problem: examining choice behaviour and probability judgments. Thinking and Reasoning, 9(1), 67-90.

Friedman, D. (1998). Monty Hall's three doors: construction and deconstruction of a choice anomaly. American Economic Review, 88(4), 933-946.

Granberg, D. (1999). Cross-cultural comparison of responses to the Monte Hall dilemma. Social Behavior and Personality, 27(4), 431-438.

Granberg, D., \& Brown, T. A. (1995). The Monty Hall Dilemma. Personality and Social Psychology Bulletin, 21(7), 711-723.

Guston, D. H. (1999). Stabilizing the boundary between politics and science: the role of the Office of Technology Transfer as a boundary organization. Social Studies of Science, 29(1), 87-112.

Guston, D. H. (2001). Boundary organizations in environmental policy and science: an introduction. Science, Technology, and Human Values, 26(4), 399-408.

Harvey, N., \& Fischer, I. (1997). Taking advice: accepting help, improving judgment, and sharing responsibility. Organizational Behavior and Human Decision Processes, 70(2), 117-133.

Hertwig, R., \& Ortmann, A. (2001). Experimental practices in economics: A methodological challenge for psychologists? Behavioral and Brain Sciences, 24(3), 383-403.

Kahneman, D., Knetch, J., \& Tversky, A. (1990). Experimental tests of the endowment effect and the Coase theorem. Journal of Political Economy, 98, 1325-1348.

Kahneman, D., \& Tversky, A. (1979). Prospect theory: an analysis of decision under risk. Econometrica, 47, 263-291.

Krauss, S., \& Wang, X. T. (2003). The psychology of the Monte Hall problem: discovering psychological mechanisms for solving a tenacious brain teaser. Journal of Experimental Psychology: General, 132(1), 3-22.

Laffont, J., \& Martimort, D. (2002). The theory of incentives. Princeton, NJ: Princeton University Press.

Lemos, M. C., Finan, T., Fox, R., Nelson, D., \& Tucker, J. (2000). The use of seasonal climate forecasting in policy-making: lessons from northeast Brazil. Tuscon: University of Arizona.

Patt, A. G. (2001). Understanding uncertainty: forecasting seasonal climate for farmers in Zimbabwe. Risk Decision and Policy, 6, 105-119.

Robinson, S. L. (1996). Trust and breach of the psychological contract. Administrative Science Quarterly, 41, 574-599.

Slembeck, T., \& Tyran, J.-R. (2004). Do institutions promote rationality? An experimental study of the three-door anomaly. Journal of Economic Behavior and Organization, 54, 337-350.

Slovic, P. (1966). Cue-consistency and cue-utilization in judgment. The American Journal of Psychology, 79, 427-434.

Sniezek, J., \& Buckley, T. (1995). Cueing and cognitive conflict in judge-adviser decision making. Organizational Behavior and Human Decision Processes, 62(2), 159-174. 
Sniezek, J., Schrah, G. E., \& Dalal, R. (2004). Improving judgment with prepaid expert advice. Journal of Behavioral Decision Making, 17, 173-190.

Teas, R. K., \& Agarwal, S. (2000). The effects of extrinsic product cues on consumers' perceptions of quality, sacrifice, and value. Journal of the Academy of Marketing Science, 28, 278-290.

Thaler, R., \& Johnson, E. J. (1990). Gambling with the house money and trying to break even: the effects of prior outcomes on risky choice. Management Science, 36, 643-660.

Tubau, E., \& Alonso, D. (2003). Overcoming illusory inferences in a probabilistic counterintuitive problem: the role of explicit representations. Memory and Cognition, 31(4), 596-607.

Yaniv, I. (2004a). The benefits of additional information. Current Directions in Psychological Science, 13(2), 75-78.

Yaniv, I. (2004b). Receiving other people's advice: influence and benefit. Organizational Behavior and Human Decision Processes, 93, 1-13.

Yaniv, I., \& Kleinberger, E. (2000). Advice taking in decision making: egocentric discounting and reputation formation. Organizational Behavior and Human Decision Processes, 83(2), 260-281.

\section{Author Note}

This research was made possible by funding from the US National Oceanic and Atmospheric Administration, Office of Global Programs (NOAA/OGP), Awards No. NA16GP2577 and BCS-0004236, and through NOAA/OGP's support of the Knowledge Systems for Sustainable Development Project based at Harvard University. We would like to thank Richard Zeckhauser, Max Bazerman, and William Clark for assistance developing the experiment, Pablo Suarez and Chiedza Gwata for assistance with the experiment administration, Eric Macklin and Bill Simpson for assistance with statistical analyses, and three anonymous reviewers for helpful comments. We would also like to remember one of the four young Zimbabwean men who helped us as advisers in the field experiment, who died later that year, leaving behind a wife, who this year has also grown ill with similar symptoms, and two young children.

Figure Captions

Figure 1. Switching rates in round 1 in the control, simple advice, aligned incentives and prepayment conditions, with the prepayment condition results reported separately for those who purchased the advice and those who did not. Error bars represent 1 standard error.

Figure 2. Switching rates in round 2 by round 1 experience in the control, simple advice, aligned incentives and prepayment conditions. (Prepayment condition results restricted to those participants who paid for advice.) Error bars represent 1 standard error.

Figure 3. Differences in the proportions of participants who switched in rounds 1 and 2 (round $2 P$-round $1 P$ ) by round 1 experience in the control, simple advice, aligned incentives and prepayment conditions. (Prepayment condition results restricted to those participants who paid for advice.) Error bars represent 1 standard error. 